\title{
Massive Hemothorax in A Pregnant Patient With Neurofibromatosis Type 1
}

\section{Kumiko Hashimoto ( $\square$ 935hashimoto@gmail.com )}

Toyohashi municipal hospital https://orcid.org/0000-0002-8523-2171

\section{Yuji Nomata}

Toyohashi minicipal hospital

\section{Takayuki Fukui}

Nagoya university

\section{Akira Takada}

Toyohashi municipal hospital

\section{Kunio Narita}

Toyohashi municipal hospital

\section{Case report}

Keywords: Hemothorax, Pregnancy, Emergency, Embolism

Posted Date: September 23rd, 2020

DOI: https://doi.org/10.21203/rs.3.rs-75605/v1

License: (c) (1) This work is licensed under a Creative Commons Attribution 4.0 International License. Read Full License 


\section{Abstract}

Background: Spontaneous hemothorax in a patient with neurofibromatosis type 1 is a less common though lethal complication, and there is no report which occurred in the pregnant period. We here present the first case of hemothorax in a neurofibromatosis type 1 patient during pregnancy and discus on the difficulty in diagnosis and treatment choice.

Case Presentation: A 39-year-old female at 34 weeks gestation presented with sudden left back pain and dyspnea. Chest radiography revealed massive left pleural effusion. Although the patient was hemodynamically stable, the fetus was in a critical condition. Emergency cesarean section and endovascular coil embolization of the aneurysm of the intercostal artery were performed successfully. The patient's condition improved immediately, and she was discharged 1 month later.

Conclusions: A neurofibromatosis type 1 patients have the potential to develop massive hemothorax without gross lesions, especially late in pregnancy and postpartum.

\section{Background}

Spontaneous hemothorax is rare, and case reports on this condition are limited [1]. Physiological changes in the perinatal period are thought to be precipitating factors for hemothorax in patients with pulmonary arteriovenous malformation and hereditary telangiectasia [2]. However, regarding patients with neurofibromatosis type 1 (NF1), there have been only three reports of cases, all of which occurred in the postpartum period. Here, we present the first case of hemothorax during pregnancy in a patient with NF1.

\section{Case Report}

A 39-year-old female at 34 weeks gestation presented with sudden left back pain and dyspnea. She had been diagnosed with NF1 over 20 years prior. She had no family history of NF1 and no history of chest trauma, prior surgery, or catheter insertion. She was on medication for gestational hypertension.

On arrival, she was conscious with a blood pressure of $110 / 90 \mathrm{mmHg}$ and a pulse rate of $90 \mathrm{beats} / \mathrm{min}$. Her hemoglobin level was $10 \mathrm{~g} / \mathrm{dL}$ and oxygen saturation was $97 \%$ with $10 \mathrm{~L} / \mathrm{min}$ oxygen delivered via mask. Chest radiography revealed a massive left pleural effusion with left-to-right mediastinal shift (Fig. 1). Chest computed tomography (CT) revealed a left pleural effusion and irregularities of the left 10th intercostal artery (Fig. 2). We suspected hemothorax because the CT value of the effusion was 45 Hounsfield units. Furthermore, CT findings also implied intrathoracic dural ectasia close to the artery. A chest tube was inserted immediately; however, only $400 \mathrm{~mL}$ of old blood was removed, and no further significant discharge was collected though the pleural effusion remained. The patient was hemodynamically stable following fluid resuscitation; however, the fetal heart rate was decreasing, and the fetus was in a critical condition. After obstetric consultation, we performed an immediate caesarean section under general anesthesia; the operative time was 40 minutes and the volume of blood lost including amniotic fluid was $300 \mathrm{~mL}$. 
Selective angiography revealed irregular vessels and small aneurysms of the left 10th intercostal artery (Fig. 3a). Extravasation of contrast agent could not be detected. Therefore, we embolized the irregularly shaped portion of the artery.

The distal site was embolized using 20 micro coils: 6 Orbit Galaxy (Codman \& Shurtleff, Raynham, MA), 6 Tornado Embolization Coils (Cook Medical, Bloomington, IN), 6 Azur Embolization System CX18 (MicroVention Inc, Aliso Viejo, CA), and 2 Interlocking Detachable Coils (Boston Scientific Corporation, Marlborough, MA). The proximal site was occluded using 3 Orbit Galaxy Microcoils. The artery and aneurysms were all embolized successfully. The duration from hospitalization to completion of treatment was about 4 hours (Fig. 3b).

The patient's condition improved postoperatively. Her blood pressure after caesarean section was $90 / 60 \mathrm{mmHg}$. After embolization, it increased to $160 / 80 \mathrm{mmHg}$. Although the intensive treatment saved her life, the fetus did not survive. Twenty days after embolization, left pleural cavity decortication was performed and we removed about $2500 \mathrm{~g}$ of hematoma; the constriction of the left lung was released. At a month post embolization, the patient was discharged in good condition. No further aneurysm or irregular arteries were found on full-body contrast-enhanced CT before discharge. No symptoms of recurrence were reported during the next 10 months, and we followed up with CT every 6 months.

\section{Discussion And Conclusions}

The autosomal dominant genetic disease NF1 affects approximately 1 in 3,000 births [3]. Patients often suffer from malignant neoplasms and various other comorbidities [4-6], thus requiring continuous medical support. Vasculopathy is a less common though lethal complication with an incidence of only $3.6 \%$ [7]. The occurrence of hemothorax in the perinatal period has been reported in only three cases of NF1, all postpartum $[5,6]$. We report, to our knowledge, the first case of massive hemothorax in a pregnant patient with NF1.

In small arteries such as the intercostal artery, invasion by neurofibroma causes intimal thinning of the media, elastic fragmentation, and aneurismal dilatation, which are influenced by the fragile nature of the vascular tissue [8]. Vascular lesions of small arteries are often asymptomatic and thus may go undetected before lethal complications occur.

Hemothorax develops when other factors, such as tumors and aneurysms, overlap with vascular vulnerability [4]. Furthermore, pregnancy might be considered a precipitating factor for hemothorax in patients with NF1. Pregnancy exacerbates NF1 itself [5.6]; furthermore, increased intrathoracic pressure, blood volume, and cardiac output lead to increased blood pressure. Elevated steroid hormone levels also increase vascular fragility.

Blood volume increases in late pregnancy, so hemorrhagic shock may be overlooked. However, the fetus is directly affected by decreased blood flow. In this case, the patient was hemodynamically stable because bleeding was restricted by a massive hematoma. We decided to perform emergency cesarean 
section with adequate infusion management because there was a possibility of saving both the patient and the fetus. If the mother's general condition had been unstable owing to persistent bleeding, endovascular coil embolization would have been performed first.

There have been several treatments reported for hemothorax, including conservative treatment with drainage, thoracotomy, and endovascular coil embolization [3]. In this case, the bleeding site was detected on contrast-enhanced CT; therefore, endovascular treatment was feasible. Surgical repair would not have been safe as the field of view would have been obscured by the massive hematoma and dural ectasia. Furthermore, the proximity of the site of bleeding to the dural ectasia meant that there was a risk of perforation during the procedure. In cases where the site of bleeding extends further to the proximal side, intrathoracic surgical repair is impossible. Therefore, considering the fragility of the artery, the site of bleeding, and the presence of dural ectasia, surgical repair would have been extremely risky.

This case demonstrates that, even in cases without gross lesions such as tumors and aneurysms, patients with NF1 patients have the potential to develop massive hemothorax during the perinatal period. Since this outcome is difficult to prevent, strict hospitalization or blood pressure control, especially late in pregnancy and postpartum, should be considered in these patients. When massive hemothorax does occur, immediate endovascular treatment and fetal lifesaving should be instituted.

\section{Abbreviations}

CT Computed tomography

NF1 neurofibromatosis type 1

\section{Declarations}

Ethics approval and consent to participate: Not applicable

Consent for publication: Yes, we have consent from the patient.

Availability of data and materials: the data that support the findings of this report are available from the Toyohashi municipal hospital. The author is available it upon reasonable request.

Competing interests: the authors declare no conflicts of interest in association with this study.

\section{Funding statement: None}

Author's contributions: All authors read and approved the final manuscript.

$\mathrm{KH}$ performed the treatment decision and wrote this report.

NY contributed to patient management during hospitalization. 
TF advised the treatment and elaborated of this case report.

AT contributed to endovascular treatment.

$\mathrm{KN}$ is the main person responsible for treatment.

Acknowledgments: None

\section{References}

1. Davide P, Nikolaos P, Jonathan P, Gvinianidze L, lqbal Y, Lawrence DR. Etiology and management of spontaneous haemothorax. J Thorac Dis. 2015;7:520-6.

2. Clare ML, Francis W, John S. Massive hemothorax due to enlarging arteriovenous fistula in pregnancy. Chest. 1992;101:1452-4.

3. Pantazopoulos I, Papazoglow G, Strataki K, Chalkias A. Spontaneous hemothorax complicating von recklinghausen disease: case report and treatment algorithm. J Emerg Med. 2019;58(2):e63-6.

4. Miura T, Kawano Y, Chujo M, Miyawaki M, Mori H, Kawahara K. Spontaneous hemothorax in patients with von recklinghausen's disease. Jpn J Thorac Cardiovasc Surg. 2010;10:128-30.

5. Brady DB, Bolan JC. Neurofibromatosis and spontaneous hemothorax in pregnancy: Two case reports. Obstet Gynecol. 1984;63:35-8.

6. Sathianurthy N, Govindasamy HD, Seevalingam KK, Paramasvaran G, Ramasamy U. Spontaneous massive haemothorax in the peri-partum period of an undiagnosed neurofibromatosis type 1 patient - a surgical perspective. Med J Malaysia. 2019;74:99-101.

7. Brasfield RD, Gupta TKD. Von recklinghausen's disease: a clinicopathological study. Ann Surg. 1972;175:86-104.

8. Greene JF Jr, Fitzwater JE, Burgess J. Arterial lesions associated with neurofibromatosis. Am J Clin Pathol. 1974:62:481-7.

\section{Figures}




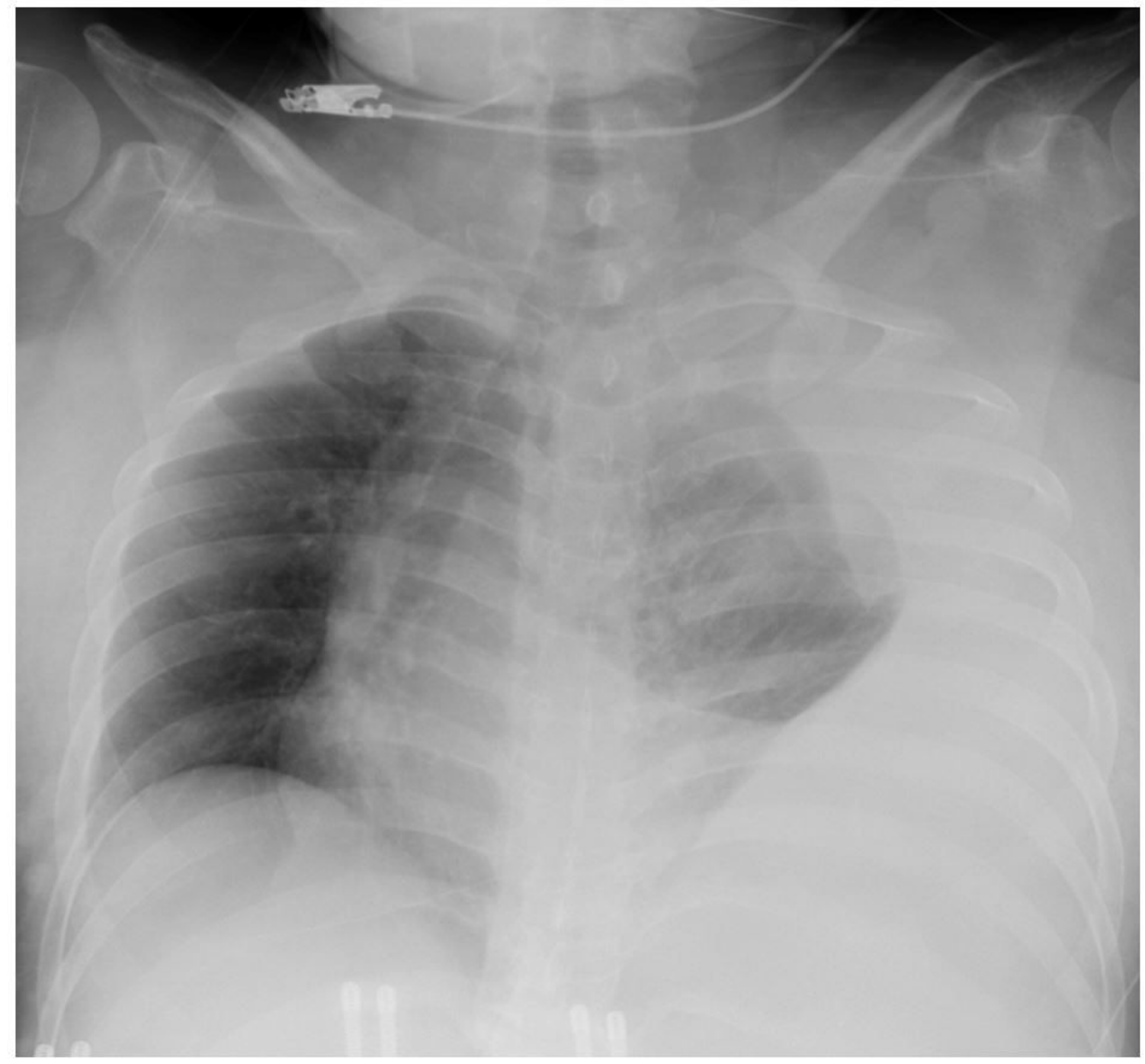

\section{Figure 1}

Frontal chest radiograph showed a massive left pleural fluid collection with a mediastinal shift to the right 


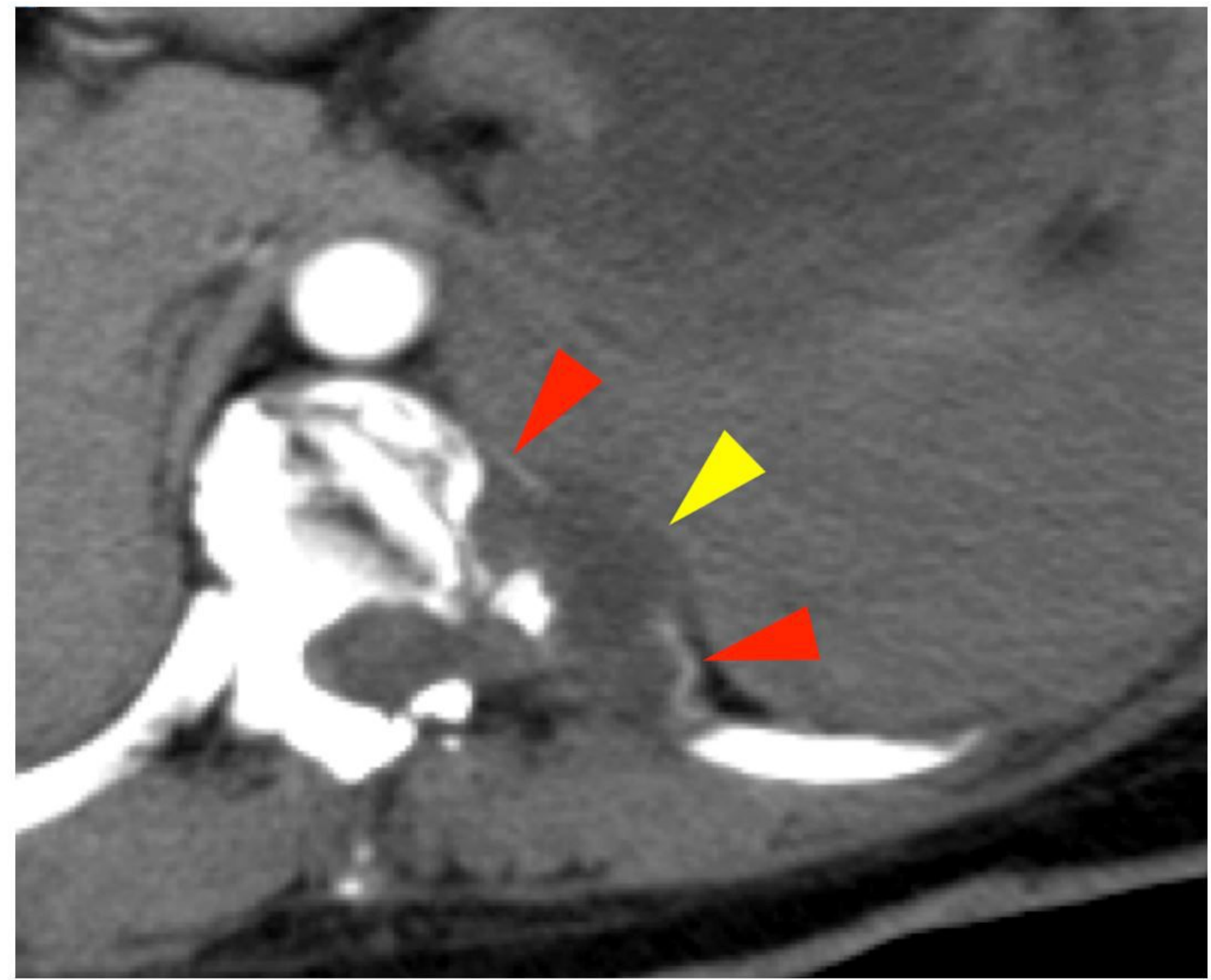

Figure 2

Chest computed tomography showed a massive hemothorax and an irregularly shaped left 10th intercostal artery (red arrow). Intrathoracic dural ectasia was observed close to the artery (yellow arrow). 


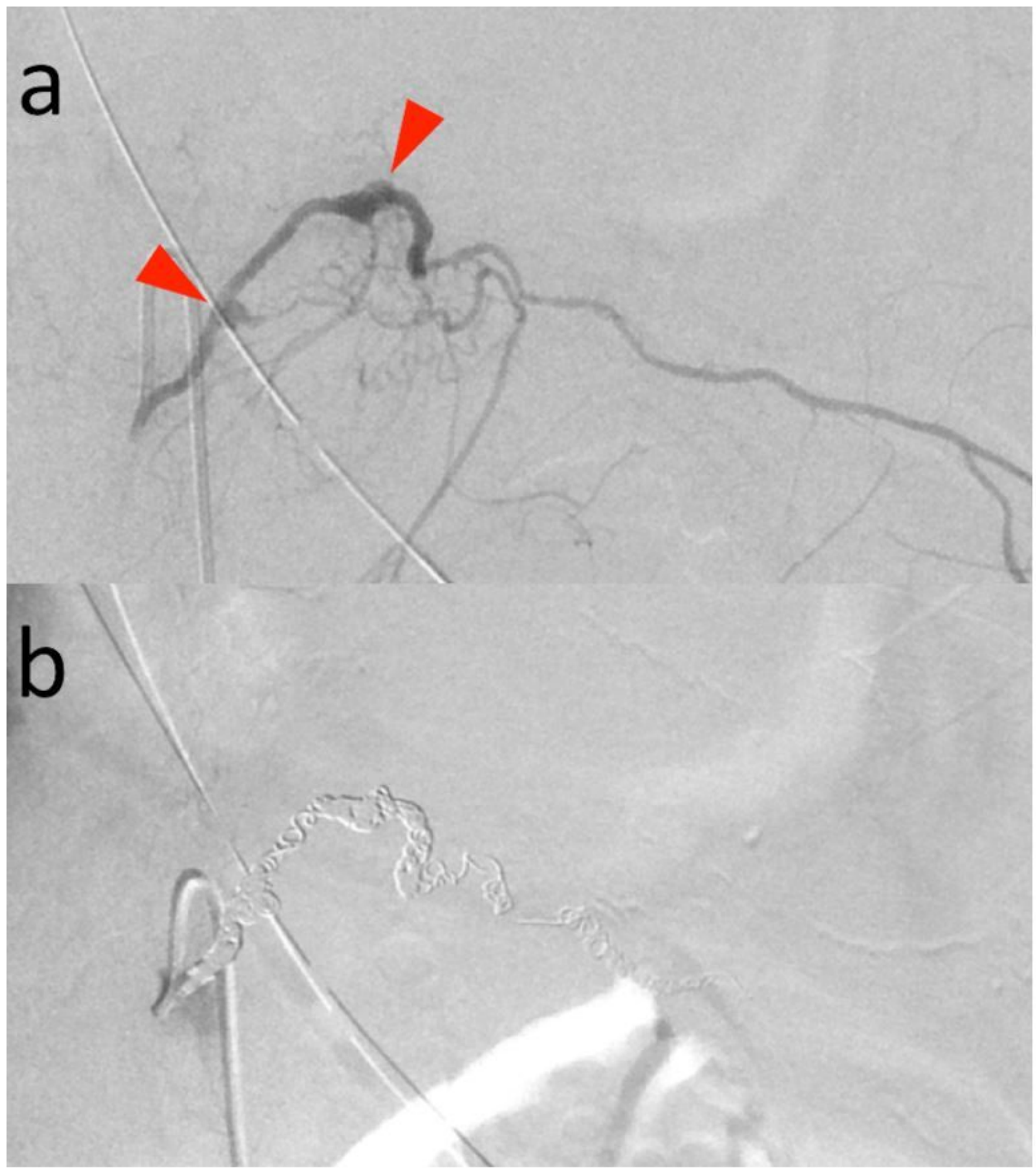

\section{Figure 3}

(a) Selective left 10th intercostal arteriography demonstrated irregular vessels and small aneurysms (red arrows). Extravasation of contrast agent was not detected. (b) Left 10th intercostal arteriography after coil embolization showed complete occlusion of the aneurysms. 